\title{
ANALYSIS AND CO-SIMULATION OF AN IEEE 802.11B WIRELESS NETWORKED CONTROL SYSTEM
}

\author{
Jeremy Colandairaj George W. Irwin ${ }^{1}$ \\ William G. Scanlon
}

\author{
School of Electrical and Electronic Engineering, Queen's \\ University Belfast, U.K.
}

\begin{abstract}
With the recent interest in closed-loop control using standard wireless networks, there is need for accurate network modelling to study the dynamic interaction between the network protocol and the control system. We present a co-simulation tool, written as a C MEX S-function within Simulink, that emulates a networked control system (NCS) with mixed-traffic stations on an IEEE 802.11b wireless network. The underlying simulation framework is explained, including both analytical and comparative validation. Detailed simulations and analysis are performed on a temperature process and a cart with an inverted pendulum to investigate the suitability of the $802.11 \mathrm{~b}$ protocol for NCS implementation. Copyright@2005 IFAC
\end{abstract}

Keywords: Networked Control Systems, Network Simulation, Wireless Networks, IEEE 802.11

\section{INTRODUCTION}

In Networked Control Systems (NCS), field devices i.e. plant, sensor, controller, and actuator are located separately but interconnected through a communications network. The major difficulty in implementing a NCS is the introduction of time delays in the control feedback loop.

In a distributed control system, plant and controller are connected by point-to-point cable, with negligible propagation delay and without contention for the use of the communication medium. On a communications network however, there is contention for bandwidth and this introduces delays in both the measurement and control signals. Such time delays may result in reduced performance or even instability.

1 Corresponding Author. Tel: (+44) (0)2890 335439; Fax: (+44) (0)2890 664265. E-Mail: g.irwin@ee.qub.ac.uk.
Analysis of these delays is therefore important to better understand the effect on the control system. Therefore the nature of the delay itself must be accurately modelled. Time delays on communication networks are not random but are dependent on various factors, for instance traffic load and the type of protocol. Poorly modelled delays can lead to inaccurate assumptions, thus producing the wrong conclusions from a stability analysis. Delay modelling using statistical distributions is insufficient for this purpose and network modelling, which exactly reproduces the effects of the network protocol is required (Colandairaj et al., 2004). Two network modelling simulators have recently been developed for the MATLAB/Simulink environment namely NCsimulator (Lian, 2001) and TrueTime (Cervin et al., 2003). However, neither supports wireless networks and use overly simplified network models. 

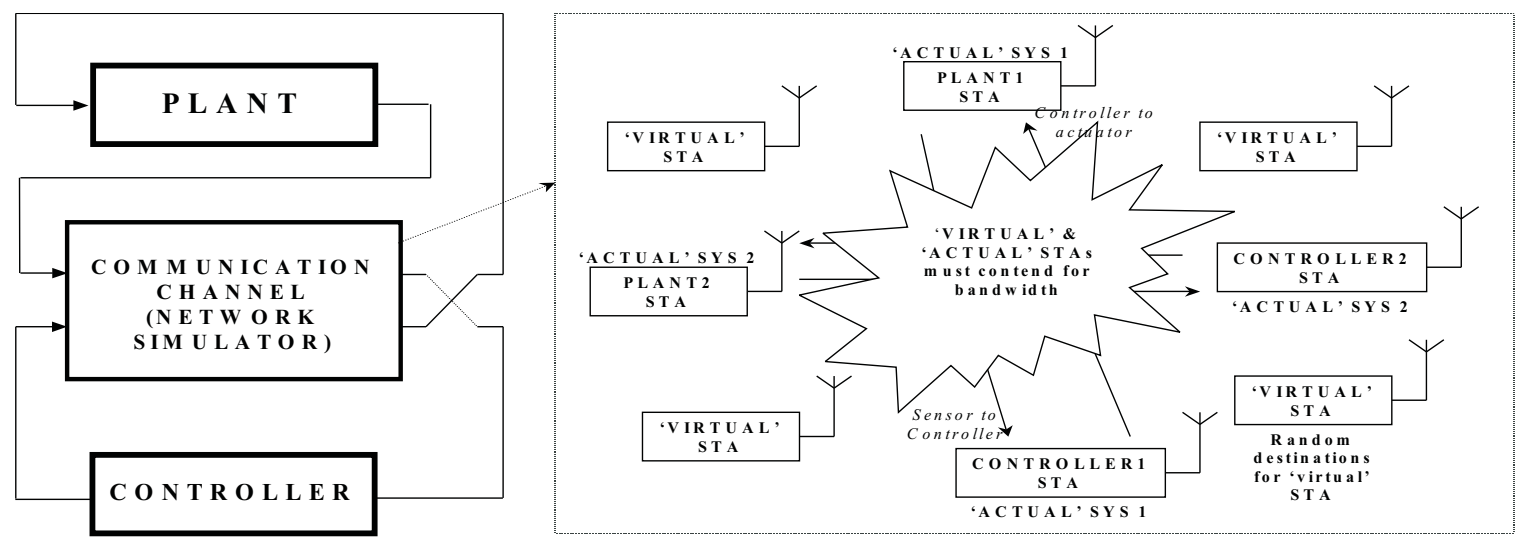

Fig. 1. Representation of control and network simulations simultaneously executed in Simulink.

The aim of this paper is to present a new network simulator based on IEEE 802.11b Wireless LAN (WLAN) and implemented as a C MEX Sfunction. The need for the simulator and design framework has been discussed in an earlier publication (Colandairaj et al., 2004). This paper presents new validation results and then applies the simulator to investigate the suitability of the standard 802.11b WLAN protocol for networked control of either a temperature process or a cart with an inverted pendulum.

The paper is organised as follows. The simulator is described in section 2 , while section 3 provides details of the validation process. The result of simulation studies from the co-simulation between network simulator and the control system case studies are then presented in section 4. Finally, in section 5 conclusions and future work are discussed.

\section{NETWORK SIMULATION FRAMEWORK}

The network simulator was based on the standard 802.11b, ad-hoc WLAN. The basic access Distributed Coordination Function (DCF) technique was chosen for its simplicity and widespread use in the domestic and commercial sectors. There is universal interest in wireless networks generally, including their potential for use in closed-loop control. Wireless adds freedom of mobility while reducing setup and maintenance costs. Possible industrial use of WLAN NCS in the avionics industry includes test bed engine monitoring and on-line aircraft monitoring (Thompson, 2004).

The reason for modelling the network within the Simulink environment is twofold: (i) it is the 'de facto' program used by academia and industry for simulation of dynamical control systems, and (ii) tools for building custom, user-defined blocks are already provided in the form of S-functions, which makes interfacing between the network and control system straightforward. An S-function is a computer language description of a Simulink block which enables the addition of user developed blocks to Simulink models. A consequence of the integration with Simulink is the ability to run the control and network simulation simultaneously.

An illustration of how the control and network simulations interact is presented in Figure 1. Here there are two types of stations. Stations representing control components are defined as control stations while those generated by the simulator to imitate other network traffic are labelled 'virtual' stations. The output from a Simulink block representing a particular process acts as input to the network simulator. When the simulator receives the control data, it is stored in a queue and has to wait until frames preceding it have been transmitted. Once it reaches the head of the queue, the station must contend with other stations intending to transmit for bandwidth on the channel. If the station wins contention and no collisions occur during this period, transmission begins. Once a transmission was completed, the contention period starts again. The frame departing from the network enters the controller block which processes the data and sends out the appropriate control action into the network. When the controller wins contention, the frame is then input to the actuator which translates the data into its corresponding input signal.

\section{VALIDATION}

In the case of co-simulation, not only must the protocol be checked for precision, but the performance of the control system must be consistent with the delays. Hence, the validation process was split into two parts: analytical validation of the network simulation and comparative validation of the co-simulation. Normalised Mean Squared Error (nMSE) was used for a numerical measure of fitness, where the measurements are normalised between \pm 1 . 


\subsection{Analytical validation of network simulation}

For the analytical validation, a simple network was constructed, simulated for 1000 s. until steady-state was reached, and then the simulation results for the frame waiting time in the queue, $W_{q}$, the total time in the network, $T_{\text {network }}$, and the mean throughput, $S$ were compared to those from queuing theory which is the theoretical study of waiting lines, expressed in mathematical terms.

Table 1. DSSS system parameters and additional parameter values

\begin{tabular}{ll}
\hline Parameter & Value \\
\hline $\mathrm{T}_{\text {SIFS }}$ & $10 \mu \mathrm{s}$ \\
$\mathrm{T}_{\text {DIFS }}$ & $50 \mu \mathrm{s}$ \\
$\mathrm{T}_{\text {slot }}$ & $20 \mu \mathrm{s}$ \\
MAC header & $272 \mathrm{bits}$ \\
PHY header & $192 \mathrm{bits}$ \\
Data frame size & $(8224+\mathrm{MAC} \&$ PHY header $)$ bits \\
ACK frame size & $(112+$ PHY header $)$ bits \\
$\mathrm{CW}_{\min }$ & 32 \\
$\mathrm{CW}_{\max }$ & 1024 \\
\hline
\end{tabular}

The simple network consisted of two 'virtual' stations, with data transmission in one direction. Data was transmitted at $11 \mathrm{Mbps}$. while the PHY header (preamble and header) as well as the ACK frames were transmitted at the legacy rate of 1 Mbps for backward compatibility. The remainder of the network settings were as in Table 1 .
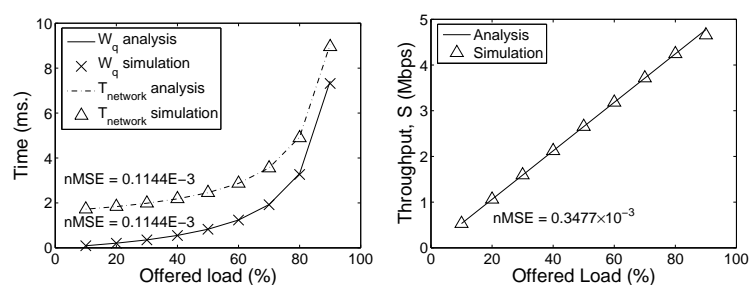

Fig. 2. Results between analysis (M/G/1 queuing theory) and simulation for $W_{q}$ and $T_{\text {network }}$ (left) and mean throughput (right)

Since the frame arrival time was Poisson distributed and the service time (time to deliver a frame from queue to destination) can be approximated by a discrete uniform distribution, an $\mathrm{M} / \mathrm{G} / 1$ queue was sufficient to model the network. Figure 2 compares the simulation results with those from queuing theory. Since the nMSE $<0.1 \%$, the simulator faithfully represents the analytical queuing theory model.

\subsection{Comparative validation of interaction between network and control system}

The sole effect of the network simulator should be to add time-varying delays to the feedback loop. To prove this, two simulations with different settings were set up; one with the network simulator and the other with pure time delays taken from the simulator output file. If no error exists in the interaction between the network simulator block and the other Simulink blocks, the responses of the control system should be identical.

The temperature process used to support the validation is described in detail in Section 4.1. The network settings were as in Table 1, with a data transmission rate of $11 \mathrm{Mbps}$. The plant was subjected to a $3^{\circ} \mathrm{C}$ step change in demanded temperature under two different loading conditions; (i) light loading which consisted of a single control system (16.4\% offered load), and (ii) saturated conditions with eight control systems running simultaneously (131.2\% offered load).

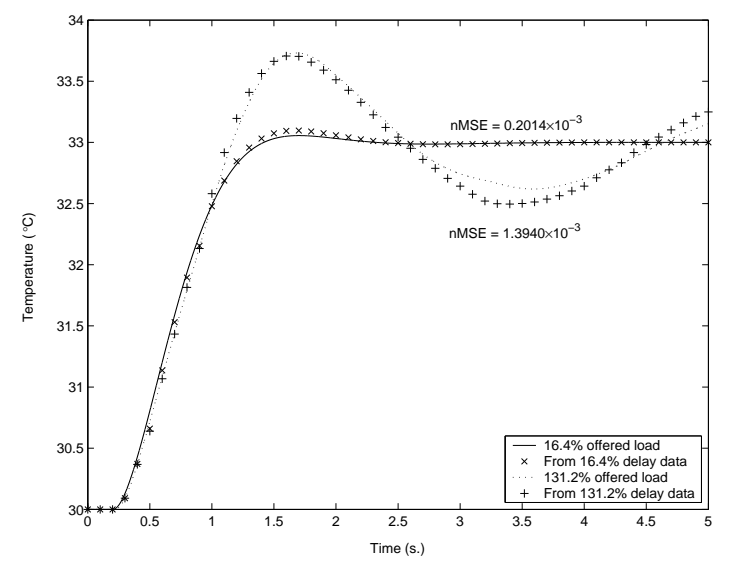

Fig. 3. Step response with the network simulator and with delays from simulator data

Knowing the magnitude of the delays and when they occurred from the output file of the simulator, the same delays were used in a separate simulation of the closed-loop temperature process with the network removed. Figure 3 shows the step responses under both simulation settings and loading conditions. The responses match closely, with the nMSE $<0.2 \%$, confirming that the dynamics of the control system remain unchanged by the network simulator except for effects of the expected network time delays.

\section{SIMULATION RESULTS AND ANALYSIS}

\subsection{Control system case studies}

Each control system on the NCS consisted of two stations on the network; the plant and controller, both in separate locations but connected by an 802.11 b WLAN. Two linear models of control systems with different dynamical properties were used to give deeper analytical insight into the impact of the network. The two different plants were sampled at an interval of $20 \mathrm{~ms}$. for computer-control with event-driven controllers. 
Temperature process (Irwin and Thompson, 1984) This well-known open-loop temperature process is described by:

$$
\dot{T}=\left[\begin{array}{cc}
0 & 1 \\
-20 & -10.2
\end{array}\right] T+\left[\begin{array}{c}
0 \\
24.8
\end{array}\right] u
$$

A discrete-time PI controller with a proportional constant, $K_{P}=0.47$ and an integral constant, $K_{I}=1.1$ was implemented. The plant is openloop stable and can be categorised as a soft realtime system (RTS). Such systems only suffer from reduced performance due to delays and remain stable as long the magnitude of the delays are not overly large.

Cart with inverted pendulum (Xiangheng and Goldsmith, 2003). The pendulum must remain upright whenever changes are made to the cart position by a force applied to the cart. The continuous-time state-space equation is given by:

$$
\left[\begin{array}{c}
\dot{x} \\
\ddot{x} \\
\dot{\theta} \\
\ddot{\theta}
\end{array}\right]=\left[\begin{array}{cccc}
0 & 1 & 0 & 0 \\
0 & -0.2 & 2.7 & 0 \\
0 & 0 & 0 & 1 \\
0 & -0.5 & 31.2 & 0
\end{array}\right]\left[\begin{array}{c}
x \\
\dot{x} \\
\theta \\
\dot{\theta}
\end{array}\right]+\left[\begin{array}{c}
0 \\
1.8 \\
0 \\
4.5
\end{array}\right] u
$$

Assuming all state variables are available, the state-feedback control matrix was:

$$
K=\left[\begin{array}{llll}
-53.555 & -30.5391 .594 & 17.565
\end{array}\right]
$$

The plant is open-loop unstable, which is more difficult to control. Such systems are termed hard RTSs and must execute within a certain deadline; delays under any circumstance causes the system to be out of control, leading to instability.

\subsection{Network setttings, assumptions and measure of performance}

All simulations used the same parameters as in Table 1, with data transmission rates as specified below. The following key assumptions were made: The channel is error free and frames are never dropped; computational delays are negligible and are neglected; and the Hidden Terminal problem, unique to wireless LANs, is ignored as well as the effects of 'Power-Saving Mode'.

To make a quantitative comparison of performance, an ITAE performance index was defined as:

$$
I T A E=\int_{t_{0}}^{t_{f}} t|e| d t, \sum_{k=k_{0}}^{k_{f}} k\left|e_{k}\right|
$$

where $t_{0}\left(k_{0}\right)$ and $t_{f}\left(k_{f}\right)$ are the initial and final times of the evaluation period in continuous (discrete) time and $e$ is the error between the actual and reference trajectories. A normalised ITAE was used for better comparison between the undelayed and delayed cases,

$$
I T A E_{N}=\frac{I T A E}{I T A E_{I}}
$$

where $I T A E_{I}$ represents the performance without delays from the network. The bigger the value of $I T A E_{N}$, the further the performance departs from the desired undelayed performance.

\subsection{NCS with a single control system}

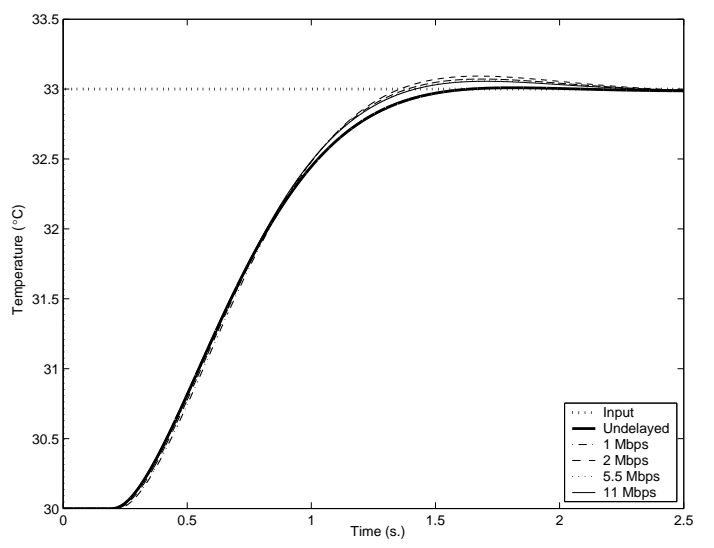

Fig. 4. Step responses for the temperature process with different data transmission rates

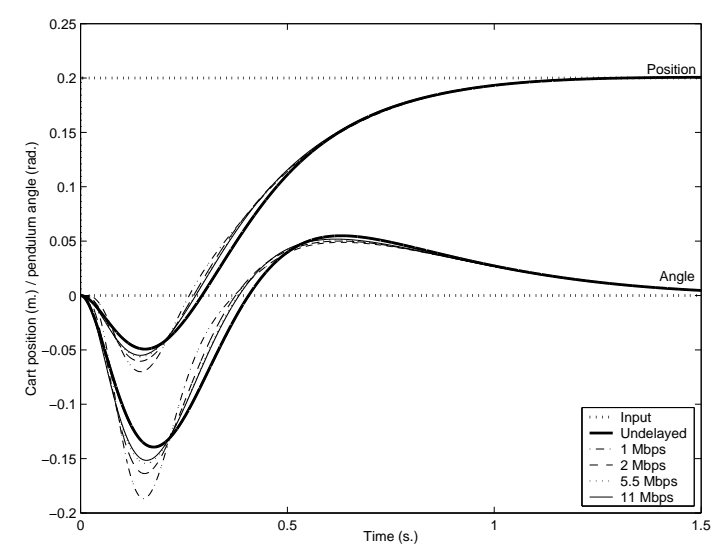

Fig. 5. Step responses of cart position and pendulum angle with different data transmission rates

Due to the network protocol, time delays exist even for a single control system with no disturbing stations. Although the network operates at a raw data rate of 11 Mbps., it can scale back to 5.5, then 2, then 1 Mbps.; depending on congestion. Table 2 shows the percentage of offered load taken up by a single control system on the network, at different rates.

Table 2. Percentage of offered load produced by a single control system on the network

\begin{tabular}{cc}
\hline Transmission rate (Mbps.) & Offered load $(\%)$ \\
\hline 1 & $94.47 \%$ \\
2 & $51.10 \%$ \\
5.5 & $24.10 \%$ \\
11 & $16.40 \%$ \\
\hline
\end{tabular}


Table 3. Control performances for a single control system with a step input

\begin{tabular}{ccc}
\hline & \multicolumn{2}{c}{ Divergence from $I T A E_{I}(\%)$} \\
Tx. rate & Temperature process & Inverted pendulum \\
(Mbps.) & $\left(I T A E_{I}=9.5653 \times 10^{4}\right)$ & $\left(I T A E_{I}=6.6891 \times 10^{3}\right)$ \\
\hline 1 & 6.72 & 3.61 \\
2 & 2.97 & 3.08 \\
5.5 & 1.02 & 2.91 \\
11 & 0.92 & 2.53 \\
\hline
\end{tabular}

A $3{ }^{\circ} \mathrm{C}$ rise in temperature was applied to the temperature process while a force causing a displacement of $0.2 \mathrm{~m}$. was applied to the cart. The control performance results are presented in Table 3 , while the resultant step responses are shown in Figures 4 and 5 respectively.

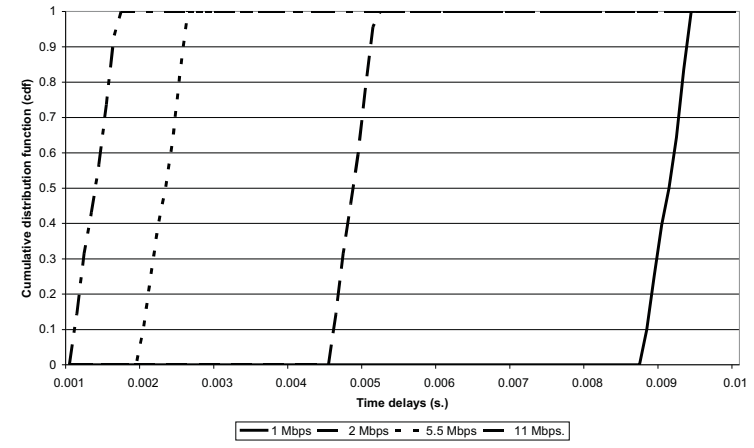

Fig. 6. Distribution of delays for a single control system under different transmission rates

It can be seen that both control systems easily maintain stability, with the temperature process particularly robust to the effects of the network. However, the cart position and angle of the pendulum overshoot more at lower transmission rates; the inverted pendulum requires a faster response from the controller than the network can provide. The distribution of the delays under different transmission rates is shown in Figure 6.

\subsection{NCS with multiple control system loading}
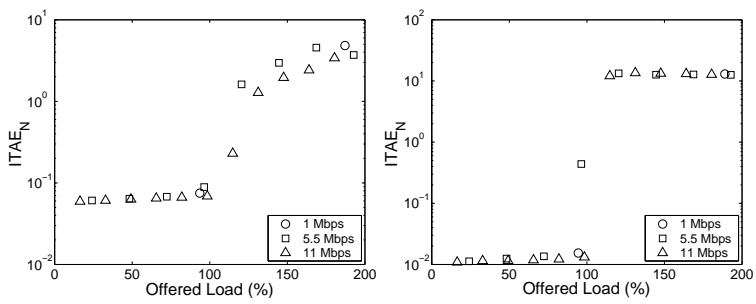

Fig. 7. Control performance vs. offered load, process trainer (left) and inverted pendulum (right)

The effect of adding multiple control systems onto the network can be seen in Figure 7. The $I T A E_{n}$ values shown are the average from each control system. By adding multiple control systems, stations must now contend with each other for a chance to transmit. Frame contention and collisions cause additional delays, which reduces performance. The performance remains stable when the offered load is within the bandwidth of the channel but a sharp decrease can be seen when the network enters saturation.

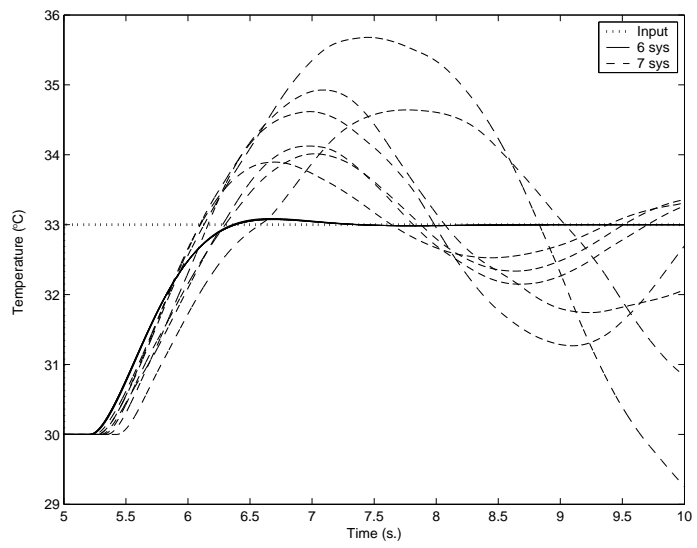

Fig. 8. Step responses of process trainer on a 11 Mbps NCS

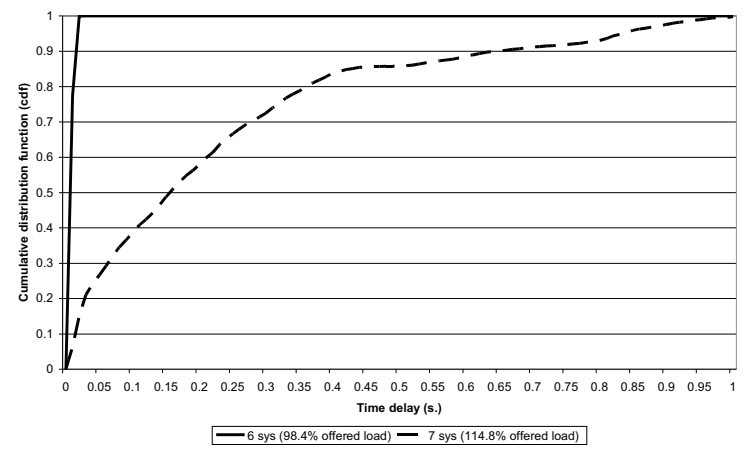

Fig. 9. Change in distribution of time delays from unsaturated to saturated network conditions

The differences in the step responses between six (98.4\% offered load) and seven (114.8\% offered load) control systems on the $11 \mathrm{Mbps}$ network for the temperature process are shown in Figure 8. The step input was delayed for 5 seconds to allow the network to reach steady-state. With 6 control systems, all remained stable with responses almost identical to the undelayed case. However, there was a great difference in delay magnitudes under saturated condition as seen in Figure 9. The channel had insufficient bandwidth to handle the offered load which caused frame buildup in the queues of the transmitting stations leading to increasing delays in the feedback loop.

Where the temperature process is robust to moderate time delays, the extra sensitivity of the inverted pendulum to delays makes the system unstable even before reaching the limitation of the bandwidth as seen in the step response in Figure 10. Therefore when designing a NCS, not only is the available network bandwidth important; the 


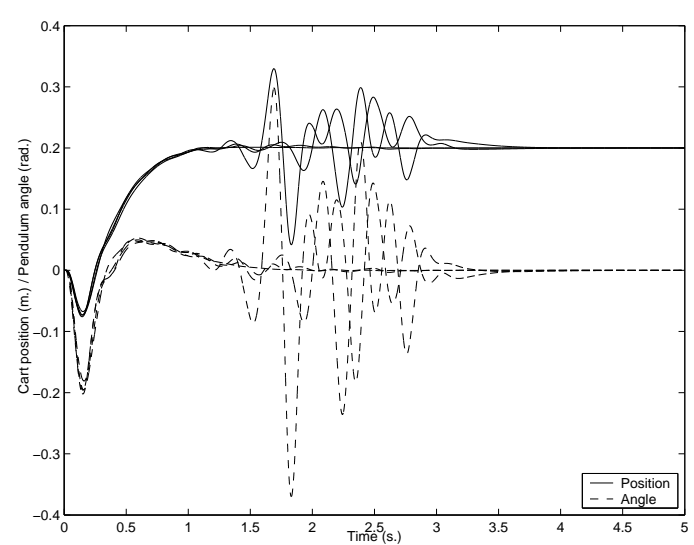

Fig. 10. Step responses of inverted pendulum on a 5.5 Mbps NCS running 4 systems (96.4\% offered load) simultaneously

type of control system needs to be considered, as the effect of the delays (nature and magnitude) on performance vary from one control system to the next.

\subsection{NCS with mixed-traffic loading}

Non-control data may also be present in a practical NCS. These take the form of alarm signals, signals for fault and data management, or even data that would be transmitted on regular LAN which could include audio/video streaming. The presence of mixed-traffic on the network was simulated using 'virtual' stations. Frame interarrival times for 'virtual' stations were modelled using negative exponential distribution centred about the average frame interarrival time of $20 \mathrm{~ms}$. similar to the plant sampling rate, while frame sizes variations are uniformly distributed between 8024 to 8424 bits; giving a mean frame size similar to the control data frame size.
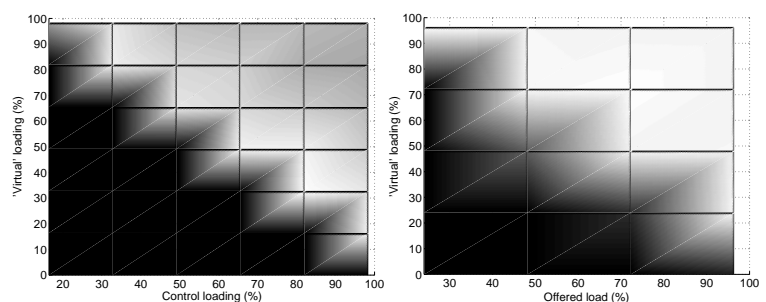

Fig. 11. Stability under various loading combinations of control and 'virtual' stations; process trainer (left) and inverted pendulum (right). Black represents stable control performance while grey and white shades show instability.

Figure 11 shows the stability under different loading combinations. For the temperature process, the system became unstable only whenever a combination of control and 'virtual' loading exceeded the maximum throughput. In the case for the inverted pendulum, the bursty nature of the traffic produced by the 'virtual' stations degrades control performance worse than the constant traffic generated by control stations, therefore causing a smaller range of stability.

\section{CONCLUDING REMARKS}

A co-simulation tool that emulates the performance of a NCS has been developed and successfully verified. From the simulation results, several key insights on the 802.11 b protocol were found. Physical transmission rates play a big part in the performance of the NCS. The faster the network, the shorter the time delays experienced by the control system. Any less than $11 \mathrm{Mbps}$. tend to cause significant performance degradation. Also, there is the limitation that, when operating without any form of priority, control and other data are treated equally during contention. Ideally for an NCS, control data should be given higher priority so that delays experienced by the control systems will be kept to a minimum.

The simulator provides a good platform for future NCS research. This will include investigations into the effects of frame droupout, protocol analysis for real-time control and new controller structures to counter the negative effects of the network.

\section{REFERENCES}

Cervin, A., D. Henriksson, B. Lincoln, J. Eker and K-E. Årzén (2003). How does control timing affect performance?. IEEE Control Systems Magazine 23(3), 16-30.

Colandairaj, J., W.G. Scanlon and G.W. Irwin (2004). Co-simulation framework for a networked control system within an IEEE $802.11 \mathrm{~b}$ wireless network. In: Proceedings of the IEE Irish Signal and Systems Conference. Belfast, U.K.. pp. 207-212.

Irwin, G.W. and S. Thompson (1984). Use of a microcomputer for classical control system design work. In: Proceedings of the IEE International Conference on Electrotechnics. Brighton, U.K.. pp. 384-88.

Lian, F-L. (2001). Analysis, Design, Modeling, and Control of Networked Control Systems. PhD thesis. Department of Mechanical Engineering. University of Michigan.

Thompson, H.A. (2004). Wireless and internet communications technologies for monitoring and control. Control Engineering Practice 12(6), 781-91.

Xiangheng, L. and A. Goldsmith (2003). Wireless communication tradeoffs in distributed control. In: Proceedings of the IEEE Conference on Decision and Control. Maui, HI, U.S.. pp. 688-94. 